\title{
Priorización de Riesgos Operacionales para un Proveedor de Tercera Parte Logística - 3PL
}

\author{
Juan C. Osorio*, Diego F. Manotas y Leonardo Rivera \\ Universidad del Valle, Facultad de Ingeniería, Escuela de Ingeniería Industrial, Grupo de Investigación en \\ Logística y Producción, Calle 13 No 100-00. Cali, Colombia. (e-mail: juan.osorio@correounivalle.edu.co) \\ ${ }^{*}$ Autor a quien debe ser dirigida la correspondencia
}

Recibido Nov. 22, 2016; Aceptado Ene. 18, 2017; Versión final Mar. 31, 2017, Publicado Ago. 2017

\begin{abstract}
Resumen
Se presenta una metodología para la gestión de los riesgos operacionales en una compañía proveedora de servicios logísticos, particularmente para los servicios de transporte y almacenamiento. Según informes nacionales e internacionales, estos servicios son los que más se subcontratan en la actualidad. La metodología desarrollada utiliza una lista de chequeo para la identificación de los riesgos, y la matriz de probabilidad e impacto, además del análisis jerárquico difuso (fuzzy analytic hierarchy process, AHP) para la priorización de los mismos. La metodología ha sido validada en una empresa 3PL colombiana (servicios de logística tercerizados) encontrándose que los riesgos más relevantes son delitos como robos y actos terroristas. También aparecen otros problemas tales como las huelgas y los defectos técnicos en el transporte terrestre de las mercancías y el robo de la mercancía, desastres naturales y deterioro en el almacenamiento. Esta metodología le permite a la organización emprender acciones para minimizar los riesgos que mayor impacto tienen en los resultados globales de la organización.
\end{abstract}

\section{Prioritization of Operational Risks for Third Party Logistics Suppliers - 3PL}

\begin{abstract}
A methodology for managing operational risks in a company that provides transportation services, specifically for its transportation and warehousing services is presented. According to domestic and international reports these are currently the most commonly outsourced services. The proposed methodology uses a checklist for the identification of risks, and the probability-impact matrix, besides the fuzzy analytic hierarchy process, AHP for their prioritization. The methodology was applied in a Colombian 3PL company (Third-Party Logistics) finding that the most relevant risks were crimes such as thefts and terrorist acts. It also appears other problems such as road blockages caused by protests, technical problems in ground transportation operations, product thefts, natural disasters, and product deterioration during storage. This methodology allows the organization to direct their actions to minimize risks that have higher global impact on the organization.
\end{abstract}

Keywords: risk management; supply chain; 3PL companies; transport; warehousing 


\section{INTRODUCCIÓN}

El riesgo es definido como una exposición a la posibilidad de impacto económico negativo, daños físicos o retrasos como consecuencia de la incertidumbre asociada con las acciones desarrolladas (Chapman and Cooper, 1983; Juttner et al, 2003 citados por Lam et al., 2015). Generalmente cada actividad de negocio está conectada con los riesgos (Pfohl et al., 2011). El riesgo no puede ser eliminado, por lo tanto las organizaciones necesitan administrar todos los factores que incrementan y reducen estos riesgos de manera que logren obtener ventajas estratégicas al mínimo costo (Borghesi y Gaudenzi, 2013). La gestión del riesgo se ha convertido en la clave para evitar las pérdidas del negocio. En el contexto de la gestión del riesgo en las cadenas de suministro (SCRM por sus siglas en inglés) los eventos son caracterizados por su probabilidad de ocurrencia y sus consecuencias relacionadas dentro de la cadena (Heckmann et al., 2015). De acuerdo con (Heckmann et al., 2015) el riesgo de la cadena de suministro es la pérdida potencial de una cadena de suministro en términos de sus valores objetivo de eficiencia y eficacia, debidos a la evolución incierta de las características de la cadena cuando ocurren eventos detonantes.

La gestión de los riesgos en la cadena de suministro ha emergido como un área importante de investigación en el campo de la gestión de cadenas de suministro (Aqlan y Lam, 2015). Adicionalmente, es un tema de mucho interés para los encargados de la gestión, presentándose de esta manera como una temática importante no sólo desde la perspectiva académica sino también desde la práctica empresarial (Colicchia y Strozzi, 2012). De hecho, hablar de riesgos sin involucrar a las compañías puede ser poco efectivo a la hora de considerar la contribución de estos trabajos en el desarrollo económico de las regiones.

El riesgo corporativo puede venir de muchas fuentes, algunas relacionadas con los procesos internos de producción y distribución y otras veces de afuera. Hay muchas formas de categorizar los tipos de riesgo, pero quizás los más relevantes en términos de la cadena de suministro son: Riesgos externos a la empresa pero internos a la cadena y externos a la cadena (Manners, 2014). Tang (2006) concluye que existen dos tipos de riesgos en la cadena de suministro; riesgos operacionales y riesgos disruptivos. Los riesgos operacionales hacen referencia a la incertidumbre inherente sobre lo que pasa diariamente en las operaciones, como la incertidumbre en la demanda del consumidor, incertidumbre en el suministro e incertidumbre sobre los costos. Por su parte los riesgos disruptivos son interrupciones mayores causadas por desastres naturales o desastres ocasionados por el hombre o crisis económicas. En la mayoría de los casos, el impacto en los negocios asociado a los riesgos disruptivos es mucho mayor que el de los riesgos operacionales (Tang, 2006). Sin embargo, aunque los riegos operacionales tienen un impacto pequeño relativo en la cadena de suministro, si no se Atienden correcta y oportunamente, el riesgo se amplificará a lo largo de la cadena (Chan y Chen; 2007).

El riesgo operacional se refiere a la posibilidad de que eventos inesperados ocurran como consecuencia de alteraciones en el normal funcionamiento e incluye todas las cosas que pueden pasar en las actividades del día a día (Bolancé et al., 2012). El riesgo operacional tiene un amplio número de definiciones que difieren principalmente en detalles y énfasis. Aunque ha habido muchas discusiones alrededor, existe consenso respecto a que como mínimo incluye las interrupciones o fallas relacionadas con la gente, procesos internos, tecnología o consecuencias de procesos externos. El riesgo operacional existe cuando cualquier actividad ocurre, sea que se administre o no (Kenett y Raanan., 2011).

Si bien, dependiendo de los autores, se habla de fases tales como la identificación, evaluación, priorización, modelamiento, mitigación y monitoreo entre otros; hay algunas fases en las que coinciden más autores, entre ellos (Berenji y Anantharaman, 2011; Elmsalmi y Hachicha, 2013; Giannakis y Louis, 2011; Mand et al., 2013; Olson y Wu, 2010) y es a partir de dicha coincidencia que se presenta en la figura 1 una propuesta que recoge los elementos más importantes de acuerdo con la revisión de la literatura y que serán consideradas en adelante.
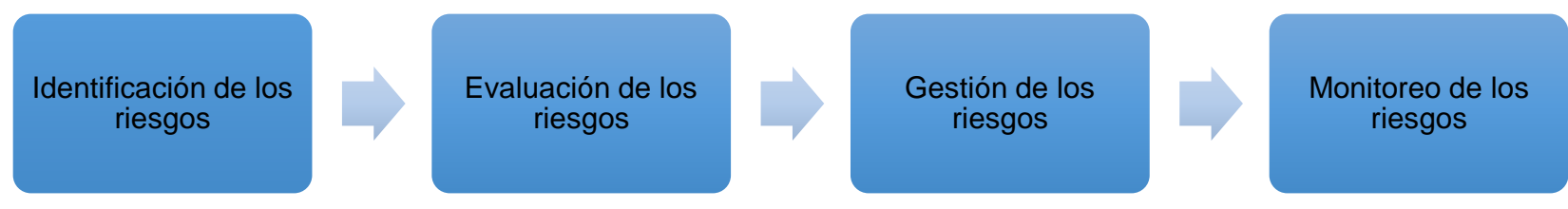

Fig. 1: Fases en un sistema de gestión de riesgos para la cadena de suministro (Manotas et al., 2016) 


\section{Identificación de los riesgos}

El primer paso en la gestión del riesgo es identificar las fuentes del riesgo. Las empresas necesitan conocer todas las posibles amenazas sistemáticamente. Los riesgos pueden encontrarse en diferentes aspectos, tanto en operaciones internas como externas. En las cadenas logísticas, la probabilidad de exposición al riesgo es más alta que en otras actividades.

La identificación de los riesgos es un importante primer paso en cualquier esfuerzo de gestión de riesgos (Singhal et al., 2011). De acuerdo con la norma ISO 31000 la identificación de los riesgos es el proceso de encontrar, reconocer y describir los riesgos e involucra la identificación de las fuentes de los riesgos, los eventos, sus causas y sus potenciales consecuencias; también, que puede involucrar datos históricos, análisis teóricos, opiniones de expertos y necesidades de las partes interesadas. Hay muchas formas para identificar y categorizar los riesgos, cada organización tiene su propia forma de desarrollar su registro de riesgos: una lista con los riesgos y su importancia. La categorización de los riesgos tiene razones importantes para la organización, algunas de las cuales pueden ser para ayudar a comprenderlos y priorizar las inversiones para mitigarlos (Sodhi y Tang, 2012). Entre las técnicas que ayudan a identificar los riesgos se cuentan: mapeo de la cadena de suministro, listas de chequeo, análisis de árboles de eventos, análisis de árboles de falla, análisis de fallas y modo de defectos (FMEA) y el diagrama causa efecto de Ishikawa (Tummala y Schoenherr, 2011).

\section{Evaluación y priorización de los riesgos}

Tal como lo plantean Tummala y Schoenherr (2011), esta actividad está relacionada con la determinación de la probabilidad de cada factor de riesgo. Según los mismos autores, dichas probabilidades pueden calcularse con información objetiva o si esta información no está disponible, recurrir a información subjetiva, sustentada en los conocimientos, creencias y juicios de los involucrados, utilizando para esto técnicas como el método Delphi o paneles de expertos y de allí derivar algunas probabilidades. Por su parte Marhavilas et al. (2011) establecen que evaluar los riesgos les permite a los encargados darle una escala a su apreciación sobre la severidad de las consecuencias tanto en el corto como en el largo plazo.

La evaluación de los riesgos se realiza para definir las acciones a realizar de acuerdo con los resultados esperados de la aparición de dichos riesgos, saber en cuáles se concentran los esfuerzos y cuáles se ignoran. Según Nan et al. (2009) la cuantificación de los riesgos en todas las investigaciones pueden ser clasificadas en dos categorías: basadas en la probabilidad y estadística y basadas en los conocimientos de los expertos. La evaluación de los riesgos ayuda a los administradores a entender los impactos negativos de los eventos adversos (por ejemplo en términos de costo y mal desempeño) y la probabilidad de las consecuencias negativas (Borghesi y Gaudenzi, 2013). Cuando se habla de riesgo se plantean dos características básicas: (i) La severidad de las posibles consecuencias adversas; y (ii) La probabilidad de ocurrencia de cada una de las consecuencias. Por ello, en la mayoría de los trabajos se define el nivel de riesgo como una medida de la severidad y la probabilidad (Borghesi y Gaudenzi 2013).

De acuerdo con la revisión realizada por Manotas et al. (2016) las técnicas multicriterio aparecen como las más comúnmente usadas en este proceso. Autores como (Aggarwarl y Sharma, 2013; Badea et al., 2014; Sofyalıoğlu y Kartal, 2012; Wang et al., 2012) utilizan el AHP y el AHP difuso para priorizar los riesgos. Por tanto, se considerará la utilización de esta herramienta en la metodología planteada.

\section{Gestión de los riesgos}

Según Faisal et al. (2006) los riesgos en las cadenas de suministro pueden ser mitigados si las compañías comprenden las variables que impactan dichos riesgos. Ellos presentan los siguientes puntos como facilitadores de la mitigación de los riesgos: (1) Compartir información; (2) Agilidad en la cadena de suministro; (3) Confianza en los socios a través de la cadena; (4) Seguridad en la información; (5) Responsabilidad social y corporativa; (6) Planeación estratégica del riesgo; (7) Relaciones de colaboración con los socios de la cadena; (8) Compartir el riesgo en la cadena; y (9) Análisis continuo y evaluación de los riesgos.

Por otro lado Sofyalıoğlu y Kartal (2012) citando a Manuj y Mentzer (2008) sugieren el siguiente grupo de estrategias para gestionar el riesgo: (1) Postponement o posposición de las últimas actividades en el proceso para darle flexibilidad y retrasar algunos costos (Tang, 2006); (2) Especulación, que es lo opuesto al postponement; (3) Controlar/ compartir/ transferir el riesgo mediante integración vertical, contratos y otros; y(4) Seguridad (sistemas de seguridad para la información y los productos). Por su parte Mensah y Merkuryev (2014) proponen las siguientes estrategias para lograr una cadena de suministro resilente: (1) Producción Lean con entregas JIT y bajos inventarios; (2) Estrategias six sigma para la cadena; (3) Incrementar la flexibilidad de la cadena; y (4) Desarrollar una fuerte cultura corporativa. 


\section{Monitoreo de los riesgos}

Siguiendo la línea del mejoramiento continuo, una vez se han definido las acciones correspondientes para mitigar o eliminar los riesgos prioritarios, la organización debe continuar monitoreando sus procesos de manera que se identifiquen nuevos riesgos y que se continúen desarrollando acciones encaminadas hacia la gestión de los riesgos restantes.

Adicionalmente al sistema de gestión de riesgos en la cadena de suministro, es importante hacer referencia al tema de la tercerización logística, la cual viene siendo utilizada como una importante estrategia empresarial en el mundo, con el que las empresas logran enfocarse en sus actividades centrales y dejan otras actividades a un tercero especializado en las mismas. Dentro de las ventajas de esta estrategia se cuentan la reducción de los costos, reducción de tiempos logísticos, disminución en el tiempo de desarrollo de nuevos productos, mejoramiento en el servicio al cliente y reducción de los costos logísticos totales, entre otros (Gunasekaran et al., 2014). En la tabla 1 se muestran los beneficios asociados a la tercerización de acuerdo con los informes anuales de los años 2013 a 2015.

Tabla 1: Beneficios del outsourcing logístico: resultados de los estudios anuales 2013 - 2015 (Langley y Capgemini Consulting, 2015)

\begin{tabular}{|l|c|c|c|c|}
\hline \multicolumn{2}{|c|}{ Resultados } & 2013 & 2014 & 2015 \\
\hline \multicolumn{2}{|l|}{ Reducción en costos logísticos } & $15 \%$ & $11 \%$ & $9 \%$ \\
\hline \multicolumn{2}{|l|}{ Reducción en costos de inventarios } & $8 \%$ & $6 \%$ & $5 \%$ \\
\hline \multicolumn{2}{|l|}{ Reducción en costos fijos logísticos } & $26 \%$ & $23 \%$ & $15 \%$ \\
\hline \multirow{2}{*}{ Cumplimiento de ordenes } & $\mathrm{De}$ & $58 \%$ & $66 \%$ & $60 \%$ \\
\cline { 2 - 5 } & $\mathrm{A}$ & $65 \%$ & $68 \%$ & $68 \%$ \\
\hline \multirow{2}{*}{ Exactitud en las ordenes } & $\mathrm{De}$ & $67 \%$ & $68 \%$ & $61 \%$ \\
\cline { 2 - 5 } & $\mathrm{A}$ & $72 \%$ & $69 \%$ & $66 \%$ \\
\hline
\end{tabular}

Para lograr cadenas de suministro exitosas se requiere tener actividades logísticas exitosas, es por ello que el papel de los proveedores de tercera parte logística - 3PL ha cambiado de algunas simples tareas a un outsourcing total convirtiéndose en no solamente un proveedor de servicios logísticos sino en un proveedor estratégico de la cadena, trabajando simultáneamente con múltiples socios en la cadena de suministro (Kumar y Singh, 2012), cambiando así de la configuración básica proveedor - fabricante - consumidor a una cadena donde los proveedores 3PL son eslabones más de la misma.

Para el resto de este documento se considerarán como 3PL aquellas organizaciones que se dedican a ofrecer los servicios logísticos a las compañías para que estas se enfoquen en sus actividades fundamentales. En este proceso el oferente aprovecha sus fortalezas y experiencia en el desarrollo de dichas actividades y el comprador se beneficia de esta experiencia concentrándose en aquellas actividades que constituyen el núcleo de su negocio. De acuerdo con Langley y Capgemini Consulting (2017) estos son los servicios más importantes que ofrecen los 3PL y el porcentaje de utilización de dichos servicios: Transporte doméstico $86 \%$; Almacenamiento $66 \%$; Transporte internacional de $60 \%$; Transporte de carga $44 \%$; Corretaje de aduanas $42 \%$; Gestión y planificación del transporte 36\%; Cross-docking 34\%; Auditoria y pago de facturas de flete 32\% ; Administración de inventarios 24\%; Gestión y cumplimiento de pedidos 24\%; Logística de reversa (defectuoso, reparación, retorno) 23 \%; Embalaje, montaje y etiquetado de productos $22 \%$.

Con respecto a América Latina de acuerdo con un estudio realizado en México en el 2012 el servicio más tercerizado es el transporte de mercancías en un 74\%, seguido por el almacenamiento con un 59\%, mientras que en Argentina es este mismo servicio pero en un nivel del $78 \%$ y el almacenamiento en un 65\%. Particularmente en Colombia, de acuerdo con la Encuesta Nacional Logística del 2015 se tenía un nivel de tercerización cercano al $50 \%$ en las empresas grandes, y el servicio que más se terceriza es el transporte de mercancías en un porcentaje superior al $70 \%$. De acuerdo con estos resultados, es evidente que los principales servicios 3PL están asociados al transporte y almacenamiento, a pesar de que hay un crecimiento indiscutible en otras actividades que están aumentando su cuota en el mercado de outsourcing.

Respecto a trabajos donde se realiza gestión del riesgo asociado a empresas 3PL se pueden mencionar (Ben-Daya y Akram, 2013; Datta et al., 2013; Lam y Dai, 2015; Li y Ma, 2014; Zuñiga y Martinez, 2016) aunque debe aclararse que ninguno de ellos presenta el tema del riesgo operacional en el transporte de mercancías. 
De acuerdo con la revisión de la literatura, se presentan en la tabla 2 los principales servicios tercerizados por las organizaciones y para los cuales recurren a un proveedor 3PL. Luego se presenta una propuesta para gestionar los riesgos asociados a estas actividades en una cadena de suministro. Finalmente la propuesta será aplicada en una empresa Colombiana dedicada a las actividades de outsourcing logístico y se discutirán los resultados.

Tabla 2: Principales servicios ofrecidos por las empresas 3PLs

\begin{tabular}{|c|c|}
\hline Servicios & Referencias \\
\hline Transporte & $\begin{array}{l}\text { Bottani y Rizzi, 2006; Aguezzoul, 2014; Sink et al., } \\
1996\end{array}$ \\
\hline Distribución & $\begin{array}{l}\text { Bottani y Rizzi, 2006; Aguezzoul, 2014; Sink et al., } \\
1996\end{array}$ \\
\hline Almacenamiento & $\begin{array}{l}\text { Bottani y Rizzi, 2006; Aguezzoul, 2014; Sink et al., } \\
\text { 1996; McGinnis et al, } 1995\end{array}$ \\
\hline Administración del inventario & $\begin{array}{l}\text { Bottani y Rizzi, 2006; Aguezzoul, 2014; Sink et al., } \\
\text { 1996; Hoek, } 2001\end{array}$ \\
\hline Empaque & $\begin{array}{l}\text { Bottani y Rizzi, 2006; Aguezzoul, 2014; Sink et al., } \\
\text { 1996; Hoek, } 2001\end{array}$ \\
\hline Logística de reversa & $\begin{array}{l}\text { Bottani y Rizzi, 2006; Aguezzoul, 2014; Sink et al., } \\
1996\end{array}$ \\
\hline $\begin{array}{l}\text { Ensamble final, Configuración del producto, Asesorías } \\
\text { sobre conceptos logísticos, Cobro a clientes, entre otros }\end{array}$ & Hoek, 2001 \\
\hline Sistemas de información & Selviaridis y Spring, 2007 \\
\hline
\end{tabular}

\section{METODOLOGÍA PROPUESTA PARA LA GESTIÓN DE LOS RIESGOS EN UNA EMPRESA 3PL}

A partir de la revisión de la literatura y considerando el sistema de gestión de riesgos presentado en la figura 1 , se propone la siguiente metodología. Esta metodología se fundamenta en los elementos más importantes de la revisión de la literatura y a continuación se detallan cada una de las fases. Es importante destacar que estas fases son secuenciales y es necesario avanzar paso a paso para lograr una adecuada gestión de los riesgos operacionales en la compañía.

\section{Identificación de los riesgos}

En esta fase se considera lo que sigue: (1) Definición de las actividades: en este punto se definen las actividades asociadas a los servicios que la empresa 3PL brinda y en las cuales se realizará la identificación de los riesgos operacionales; (2) Conformación del grupo de expertos: en este grupo deben estar las personas de la compañía que tienen el conocimiento y la experiencia asociada al desarrollo de las actividades y los posibles riesgos asociados a las mismas, es fundamental la participación de expertos conocedores de la realidad empresarial y del entorno de la cadena en la cual la compañía 3PL se encuentra ubicada, por ello, la definición de estos expertos requiere un estudio detallado y completo por parte de quien se vaya a encargar de realizar la gestión de los riesgos; (3) Diseño de la encuesta: considerando que es importante no sesgar las opiniones de los expertos, se diseña una encuesta amplia y abierta para que cada uno de manera independiente responda frente a los posibles riesgos de acuerdo con las actividades definidas; (4) Aplicación de la encuesta: se aplica de manera individual para que cada uno de los entrevistados se sienta libre y tranquilo de plantear todos los posibles riesgos; y (5) Consolidación y análisis de la información: se agrupan los riesgos comunes, se definen así todos los riesgos posibles de acuerdo con las actividades y con el conocimiento y experiencia de los encuestados. Finalmente, se puede realizar una validación final con el grupo de expertos de la información consolidada que puede contribuir a identificar algún riesgo que no se haya incluido en la primera consolidación.

Priorización de los riesgos

En esta fase se considera: (1) Presentación de los riesgos consolidados: una vez consolidada la información de las encuestas, se presenta al grupo de expertos para realizar la validación final de los mismos. Si en este momento aparecen nuevos riesgos, deben ser incluidos y proceder a la evaluación; (2) Evaluación de 
la probabilidad de ocurrencia: para este caso particular, se considerará la matriz de probabilidad impacto, en este sentido, la probabilidad se evaluará de manera cualitativa considerando la siguiente escala: muy baja, baja, media, alta y muy alta; (3) Evaluación del impacto: similar a la probabilidad de ocurrencia el impacto será medido como muy bajo, bajo, medio, alto y muy alto, considerando un impacto global que reúna aspectos de impacto económico, en el producto y en las personas; (4) Construcción de la matriz: se realiza un análisis cruzado de los resultados de probabilidad de ocurrencia e impacto; (5) Definición de las zonas de análisis: considerando los riesgos identificados y su posición en la matriz se definirán tres zonas, verde o de riesgo mínimo, amarilla o riesgo medio y roja o riesgo crítico; (6) Establecimiento de los riesgos críticos: todos los riesgos ubicados en la zona roja se consideran críticos y deben ser intervenidos prioritariamente; (7) Como propuesta adicional, se considera realizar también la priorización utilizando la técnica multicriterio AHP difuso, que permitirá confrontar los riesgos identificados con los criterios definidos como más importantes para los expertos de la organización.

\section{Gestión de los Riesgos}

En esta fase se procede como sigue: (1) De acuerdo con los riesgos priorizados establecer estrategias de mitigación: considerando los riesgos críticos y las políticas organizacionales orientar estrategias hacia la mitigación o eliminación de los riesgos; (2) Definición de responsables y cronogramas: estas estrategias deben traducirse en acciones con responsables y fechas claras para que se logre alcanzar el objetivo de mitigar o eliminar el riesgo; (3) Evaluación de los resultados: realizar seguimiento al cronograma y los resultados esperados.

\section{Monitoreo de los Riesgos}

En el monitoreo se procede así: (1) Seguimiento a la matriz: una vez implementadas las estrategias de gestión, volver a evaluar los riesgos para reubicarlos en la matriz; (2) Actualización de los riesgos: revisar los riesgos identificados y actualizar la lista si se han identificado más o si existen nuevos procesos asociados a las actividades evaluadas; y (3) Retomar el ciclo: tomar otras actividades y completar el sistema para toda la organización.

\section{APLICACIÓN DE LA METODOLOGÍA PROPUESTA Y RESULTADOS}

La metodología planteada fue aplicada en una importante empresa 3PL Colombiana, la cual brinda servicios principalmente de transporte y almacenamiento a grandes compañías nacionales e internacionales ubicadas en el país. La compañía posee una flota de camiones especializados para el transporte de mercancías y cuenta también con bodegas de almacenamiento ubicadas en las principales ciudades de manera que puede ofrecer a sus clientes la movilidad y el almacenamiento de sus productos. Aunque presta otros servicios asociados a las empresas $3 \mathrm{PL}$, el mayor porcentaje de sus actividades se concentra en el transporte terrestre y en el almacenamiento. Se omite el nombre de la compañía por razones de confidencialidad.

\section{Identificación de los riesgos}

En el caso específico de las empresas 3PL se considerarán de acuerdo con lo mencionado en el apartado anterior, los riesgos operacionales de las dos principales actividades ofrecidas: Transporte y almacenamiento de mercancías. De acuerdo con la revisión realizada se encuentra que las técnicas cualitativas son las más utilizadas y están sustentadas en el conocimiento y experiencia de los actores involucrados. Se ha utilizado entonces una entrevista con expertos vinculados a empresas 3PL y se han definido los siguientes como los principales riesgos operacionales asociados a las actividades de transporte y almacenamiento de las mercancías (Tabla 3). Una vez identificados los principales riesgos, es importante definir cuáles son más críticos con el objetivo de establecer estrategias de mitigación e intervención para ellos. Para esto, es importante valorar los riesgos, tal como se presenta en el apartado siguiente.

\section{Priorización de los riesgos}

En la figura 5 se presenta la matriz de probabilidad impacto de los riesgos identificados en el punto anterior. En esta matriz se han ubicado los riesgos de acuerdo con su probabilidad de ocurrencia y el impacto que sufrirá la organización en el momento en que dicho riesgo se presente.

Se puede observar que hay tres zonas identificadas por los colores rojo, amarillo y verde. Este código de colores busca llamar la atención sobre aquellos riesgos que serían (en el momento de presentarse) más críticos para la organización. Por tanto, todos los riesgos que estén en la zona roja deben ser atendidos por la empresa y establecer planes de gestión y contingencia para los mismos. Los que se ubican en la zona amarilla, si bien no se consideran críticos, deben ser considerados una vez se haya establecido el esquema 
de gestión para los ubicados en la zona roja. Finalmente, los ubicados en la zona verde no son prioridad para la organización pero no por ello deben ser olvidados y una vez se hayan establecido las acciones y gestiones apropiadas para los de las zonas roja y amarilla, deben ser estudiados por los encargados de la empresa.

Tabla 3: Riesgos identificados en la empresa 3PL

\begin{tabular}{|c|c|}
\hline Riesgos en el transporte terrestre & Riesgos en el almacenamiento \\
\hline $\begin{array}{l}\text { Delitos, robos y actos terroristas (RT1) } \\
\text { Desastres en las vías (RT2) } \\
\text { Paros, huelgas, manifestaciones (RT3) } \\
\text { Fallas técnicas de los vehículos (RT4) } \\
\text { Incumplimiento de las normas de tránsito (RT5) } \\
\text { Impericia de los conductores (RT6) } \\
\text { Errores en los despachos y envíos a zonas equivocadas o } \\
\text { empresas equivocadas (RT7) } \\
\text { Contaminación cruzada en el transporte (RT8) } \\
\text { Demoras por inspecciones de las autoridades (RT9) } \\
\text { Mala comunicación entre el dueño y el transportador (RT10) } \\
\text { Rupturas en la cadena de frío (RT11) } \\
\text { Problemas con la documentación del vehículo (RT12) }\end{array}$ & $\begin{array}{l}\text { Robo de la mercancía (RA1) } \\
\text { Inundaciones o desastres naturales (RA2) } \\
\text { Deterioro de la mercancía almacenada (RA3) } \\
\text { Deterioro del empaque o contaminación del } \\
\text { mismo (RA4) } \\
\text { Incendios o asonadas (RA5) } \\
\text { Contaminación cruzada en el almacenamiento } \\
\text { (RA6) } \\
\text { Pérdida de control de los movimientos del } \\
\text { inventario (RA7) }\end{array}$ \\
\hline
\end{tabular}

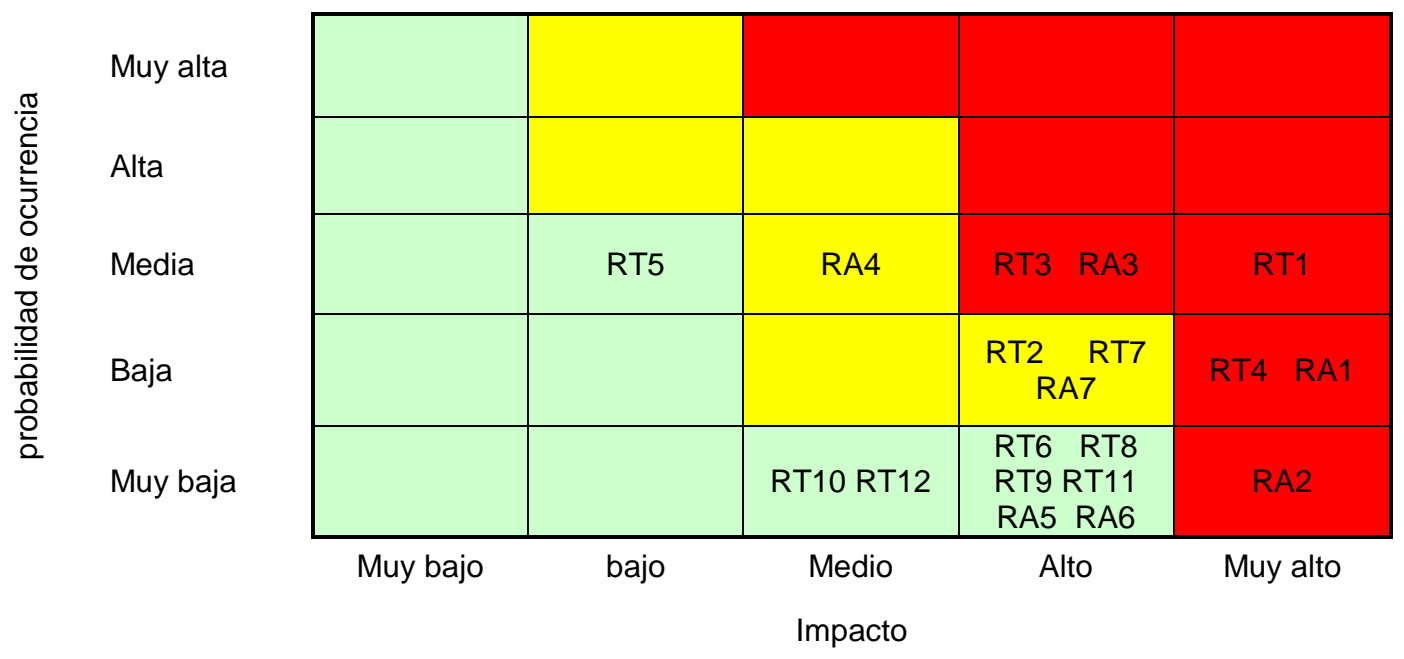

Fig. 2: Matriz probabilidad impacto para los riesgos de la empresa 3PL

Para la aplicación del AHP difuso se definieron los criterios considerados más importantes por los expertos de la organización. Posteriormente dichos criterios fueron ponderados utilizando la escala difusa presentada por (Tian y Yan, 2013) y mediante la aplicación de las comparaciones pareadas del método AHP se obtuvieron los resultados que se muestran en la tabla 4. Es de destacar, que la priorización se hizo de manera independiente para los riesgos en transporte y los riesgos en almacenamiento, y que los principales riesgos de acuerdo con esta metodología coinciden con los definidos como críticos en la matriz de la Fig. 2.

\section{Actividades de gestión y mitigación de los riesgos}

De acuerdo con estos resultados, los riesgos más importantes para la empresa 3PL en el servicio del transporte de mercancía son los riesgos RT1, RT3 y RT4 y en el servicio de Almacenamiento son RA1, RA2 y RA3; sobre estos riesgos deben enfocarse las actividades de mitigación para evitar que estos riesgos se presenten o para garantizar que en el evento de presentarse, la empresa pueda reaccionar efectiva y oportunamente de manera que las consecuencias no impacten significativamente ni a la compañía ni a la cadena de suministro involucrada. De acuerdo con los riesgos identificados para la empresa 3PL y considerando estos elementos presentados se pueden proponer las siguientes estrategias para mitigar dichos riesgos. 
Tabla 4: Priorización de los Riesgos identificados en la empresa 3PL utilizando el AHP difuso

\begin{tabular}{|c|c|c|c|}
\hline $\begin{array}{c}\text { Riesgos en el } \\
\text { transporte terrestre }\end{array}$ & $\begin{array}{c}\text { Ponderación } \\
(\%)\end{array}$ & $\begin{array}{c}\text { Riesgos en el } \\
\text { almacenamiento }\end{array}$ & $\begin{array}{c}\text { Ponderación } \\
\text { (\%) }\end{array}$ \\
\hline RT1 & 13.57 & RA1 & 21.90 \\
\hline RT2 & 7.66 & RA2 & 25.25 \\
\hline RT3 & 15.48 & RA3 & 16.45 \\
\hline RT4 & 19.04 & RA4 & 13.45 \\
\hline RT5 & 8.75 & RA5 & 6.20 \\
\hline RT6 & 3.35 & RA6 & 7.25 \\
\hline RT7 & 8.56 & RA7 & 9.50 \\
\hline RT8 & 7.07 & & \\
\hline RT9 & 4.25 & & \\
\hline RT10 & 6.37 & & \\
\hline RT11 & 3.25 & & \\
\hline RT12 & 2.65 & & \\
\hline
\end{tabular}

Respecto a los riesgos RT1, RT2, RA1 y RA2 la organización debe trabajar en su sistema de seguridad, tener asegurada la mercancía y contar con una compañía de seguridad que acompañe los transportes y vigile sus instalaciones. Además adquirir pólizas de seguro que incluyan los desastres naturales y asaltos en la vía. Respecto a RT3, RT4, RT7 y RA7 se pueden abordar desde los procedimientos internos, fortaleciendo los programas de mantenimiento preventivo para la flota, garantizando disponibilidad de flota alterna y coordinando adecuadamente su programación de despachos. Finalmente para los riesgos RA3 y RA4 de igual manera, a partir de sus procedimientos internos, estableciendo rutas de vigilancia en las instalaciones de almacenamiento y coordinando con el personal de calidad y almacenamiento el chequeo periódico de las condiciones de la mercancía. Adicionalmente, tener una política de rotación que permita sacar de las instalaciones la mercancía de mayor antigüedad podrá evitar el deterioro o la contaminación de la misma.

Está claro que estas propuestas son apenas aproximaciones y deberán ser consideradas detenidamente por las organizaciones, pero una vez se han identificado y priorizado los riesgos, la determinación de estas estrategias es mucho más efectivo y por tanto los esfuerzos serán adecuadamente orientados para garantizar una compañía 3PL más sólida y constituyéndola en un aliado importante no sólo para las actividades logísticas sino para la mitigación de los posibles riesgos en toda la cadena de suministro. Se puede incluso pensar en utilizar una herramienta multicriterio para la priorización de las acciones de gestión y mitigación de los riesgos de manera que se decida sobre aquella estrategias que abarque la mayor cantidad de riesgos y sea más costo efectiva para la organización y para la cadena.

Respecto al monitoreo, una vez definidas las acciones, estás deben desencadenar en planes de acción donde se establezca claramente los responsables, las fechas y los presupuestos involucrados de manera que se pueda realizar un control a la efectividad de las acciones y se cuente con una base de datos que permita continuar el proceso de mejoramiento de la compañía.

\section{CONCLUSIONES}

A partir de la definición de la metodología, su aplicación en la empresa 3PL Colombiana y los resultados obtenidos se puede concluir: (1) La principal característica de la metodología planteada es su sustento en el conocimiento de la organización, los procesos y la cadena de suministro en la que la empresa se encuentra. (2) Aunque ha sido aplicada para el transporte y almacenamiento, puede replicarse en todas las actividades 
logísticas brindadas por los proveedores 3PL. (3) Aunque se centra en la identificación y priorización de los riesgos, estos resultados son definitivos para la efectividad del sistema en general, pues las acciones que se deriven a continuación estarán enfocadas en los riesgos más importantes para la organización. (4) Puede ser aplicada en cualquier tipo de industria, tanto para la empresa 3PL como para los demás eslabones de la cadena de suministro. (5) La aplicación de la técnica AHP difuso para la priorización permite incluir diferentes criterios respecto a los impactos de los riegos dependiendo del tipo de organización en la que se esté realizando el proceso.

\section{REFERENCIAS}

Aggarwarl, R., y Sharma, S. Prioritization of Supply Chain Risk Assessors Using Fuzzy Analytic Hierarchy Process. 2013. International Conference on Machine Intelligence and Research Advancement, 100-104. doi:10.1109/ICMIRA.2013.26 (2013)

Aguezzoul, A. Third-party logistics selection problem: A literature review on criteria and methods. Omega, 49, 69-78 (2014)

Aqlan, F. y Lam, S. S. A Fuzzy-based Integrated Framework for Supply Chain Risk Assessment. International Journal of Production Economics, 161, 54-63 (2015)

Badea, A., Prostean, G., Goncalves, G., y Allaoui, H. Assessing Risk Factors in Collaborative Supply Chain with the Analytic Hierarchy Process (AHP). Procedia - Social and Behavioral Sciences, 124, 114-123 (2014)

Ben-Daya, M., y Akram, M. Third party logistics risk management. Proceedings of 2013 International Conference on Industrial Engineering and Systems Management, IEEE - IESM 2013, October (2013)

Berenji, H. R., y Anantharaman, R. N. Supply Chain Risk Management: Risk Assessment in Engineering and Manufacturing Industries, 2(6) (2011)

Bottani, E., y Rizzi, A. A fuzzy TOPSIS methodology to support outsourcing of logistics services. Supply Chain Management: An International Journal, 11, 294-308 (2006)

Borghesi, A., B., Gaudenzi. Risk management. How to Assess, Transfer and Communicate Critical Risks. $1^{\text {a }}$ Ed. 1-142, Springer Verlag, Italia (2013)

Colicchia, C., y Strozzi, F. Supply chain risk management: a new methodology for a systematic literature review. Supply Chain Management: An International Journal, 17(4), 403-418 (2012)

Datta, S., Samantra, C., Mahapatra, S. S., Mandal, G., y Majumdar, G. Appraisement and selection of third party logistics service providers in fuzzy environment. Benchmarking: An International Journal, 20(4), 537-548 (2013)

Elmsalmi, M., y Hachicha, W. Risks prioritization in global supply networks using MICMAC method: A real case study. International Conference on Advanced Logistics and Transport, ICALT 2013, 394-399 (2013)

Faisal, M. N., Banwet, D. K., y Shankar, R. Supply chain risk mitigation: modeling the enablers. Business Process Management Journal, 12, 535-552 (2006)

Giannakis, M., y Louis, M. A multi-agent based framework for supply chain risk management. Journal of Purchasing and Supply Management, 17(1), 23-31 (2011)

Gunasekaran, A., Irani, Z., Choy, K.-L., Filippi, L., y Papadopoulos, T. Performance Measures and Metrics in Outsourcing Decisions: A Review for Research and Applications. International Journal of Production Economics, 161, 153-166 (2014)

Heckmann, I., Comes, T., y Nickel, S. A critical review on supply chain risk - Definition, measure and modeling. Omega, 52, 119-132 (2015)

Hoek, Remko I. van. The contribution of performance measurement to the expansion of third party logistics alliances in the supply chain. International Journal of Operations \& Production Management, 21, 15-29 (2001)

Kumar, P., y Singh, R. K. A fuzzy AHP and TOPSIS methodology to evaluate 3PL in a supply chain. Journal of Modelling in Management, 7(3), 287-303 (2012)

Lam, H.Y., Choy, K.L., Ho, G.T.S., Cheng, S.W.Y., y Lee, C.K.M. A knowledge-based logistics operations planning system for mitigating risk in warehouse order fulfillment, Int. J. Production Economics, 170, 763-779 (2015)

Lam, J.S.L., y Dai, J. Developing supply chain security design of logistics service providers: An analytical network process-quality function deployment approach. International Journal of Physical Distribution \& Logistics Management, 45(7), 674-690 (2015) 
Langley, J., y Capgemini Consulting. THIRD-PARTY LOGISTICS STUDY - The State of Logistics Outsourcing. 19th Annual Study, 1-95 (2015)

Langley, J., y Capgemini Consulting. THIRD-PARTY LOGISTICS STUDY - The State of Logistics Outsourcing. 21 ${ }^{\text {th }}$ Annual Study, 1-64 (2017)

$\mathrm{Li}, \mathrm{Q}$., y Ma, J. Contract design for 4PL service supply chain and its Pareto improvement. WSEAS Transactions on Mathematics, 13, 22-30 (2014)

Mand, J.S., Singh, C. D., y Singh, R. Implementation of Critical Risk Factors in Supply Chain Management. International Journal of Management Research and business strategy, 2, 104-120 (2013)

Manotas, D.F., Osorio, J.C., y Rivera, L. Operational Risk Management in Third Party Logistics (3PL). In G. Alor-Hernández, C. Sánchez-Ramírez, y J.L. García-Alcaraz (Eds.), Handbook of Research on Managerial Strategies for Achieving Optimal Performance in Industrial Processes (Vol. i). IGI Global. U.S.A. (2016)

Marhavilas, P.K., Koulouriotis, D., y Gemeni, V. Risk analysis and assessment methodologies in the work sites: On a review, classification and comparative study of the scientific literature of the period 2000-2009. Journal of Loss Prevention in the Process Industries, 24(5), 477-523 (2011)

Mensah, P., y Merkuryev, Y. Developing a Resilient Supply Chain. Procedia - Social and Behavioral Sciences, 110, 309-319 (2014)

Nan, J., Huo, J. Z., y Liu, H. H. Supply chain purchasing risk evaluation of manufacturing enterprise based on Fuzzy-AHP method. $2^{\text {nd }}$ International Conference on Intelligent Computing Technology and Automation, ICICTA 2009, 3(70772077), 1001-1005 (2009)

Olson, D. L., y Wu, D. Enterprise Risk Management Models. $1^{a}$ Ed. 1-13. Springer Berling Heilderbeg (2010)

Pfohl, H., Gallus, P., y Thomas, D. Interpretive structural modeling of supply chain risks. International Journal of Physical Distribution \& Logistics Management, 41(9), 839-859 (2011)

Selviaridis, K., y Spring, M. Third party logistics: a literature review and research agenda. The International Journal of Logistics Management, 18, 125-150 (2007)

Sink, H.L., Jr, C.J.L., y Gibson, B.J. Buyer observations of the US third-party logistics market. International Journal of Physical Distribution \& Logistics Management, 26, 38-46 (1996)

Sodhi, M.S., y Tang, C.S. Managing Supply Chain Risk. Risk Management, 172, 3-11. Springer Verlag. New York (2012)

Sofyalıoğlu, Ç., y Kartal, B. The Selection of Global Supply Chain Risk Management Strategies by Using Fuzzy Analytical Hierarchy Process - A Case from Turkey. Procedia - Social and Behavioral Sciences, 58, 1448-1457 (2012)

Tang, C.S. Perspectives in supply chain risk management. International Journal of Production Economics, 103, 451-488 (2006)

Tian, J., y Yan, Z.F. Fuzzy Analytic Hierarchy Process for Risk Assessment to General- assembling of Satellite. Journal of Applied Research and Technology, 11(August), 568-577 (2013)

Tummala, R., y Schoenherr, T. Assessing and managing risks using the Supply Chain Risk Management Process (SCRMP). Supply Chain Management: An International Journal, 16, 474-483 (2011)

Wang, X., Chan, H. K., Yee, R. W. Y., y Diaz-Rainey, I. A two-stage fuzzy-AHP model for risk assessment of implementing green initiatives in the fashion supply chain. International Journal of Production Economics, 135(2), 595-606 (2012)

Zuñiga, R., y Martinez, C. A third-party logistics provider: To be or not to be a highly reliable organization. Journal of Business Research, 69(10), 4435-4453 (2016) 